\title{
Editorial: Integrating Oral and Systemic Health: Innovations in Transdisciplinary Science, Health Care and Policy
}

\author{
Martha Somerman ${ }^{1 *}$ and Wendy E. Mouradian ${ }^{2,3}$ \\ ${ }^{1}$ Frontiers in Dental Medicine, Chevy Chase, MD, United States, ${ }^{2}$ School of Dentistry, University of Washington, Seattle, WA, \\ United States, ${ }^{3}$ The Santa Fe Group, New York, NY, United States
}

Keywords: systems integration, dentistry, medicine, case reports, health systems

Editorial on the Research Topic

Integrating Oral and Systemic Health: Innovations in Transdisciplinary Science, Health Care and Policy

\section{OPEN ACCESS}

Edited and reviewed by:

Patrick R. Schmidlin,

University of Zurich, Switzerland

${ }^{*}$ Correspondence:

Martha Somerman

msomerman13@gmail.com

Specialty section:

This article was submitted to

Periodontics,

a section of the journa

Frontiers in Dental Medicine

Received: 12 October 2021

Accepted: 20 October 2021

Published: 08 November 2021

Citation:

Somerman M and Mouradian WE (2021) Editorial: Integrating Oral and

Systemic Health: Innovations in Transdisciplinary Science, Health Care

and Policy.

Front. Dent. Med. 2:793526. doi: 10.3389/fdmed.2021.793526

\section{INTRODUCTION}

Frontiers in Dental Medicine (FDEM) was launched in 2020, with a vision set forth by Field Chief Editor, Martha Somerman to advance the integration of medicine and dentistry at multiple levels, from basic science to clinical practice, to health policy decision making. My first assignment was to prepare a Field Grand Challenge (GC) and Special Research Topic (RT). I invited physician Wendy Mouradian to join me in crafting these documents since we share the same vision, and now more than ever, the urgency to remove silos across all disciplines to bring medicine and dentistry together to advance oral and overall health and well-being for all communities. We realize this can only be achieved through a combination of approaches including education, research, clinical practice, professional development, health care literacy, patient advocacy, health care systems and health policy development. The theme for both the Grand Challenge and Research Topic was therefore "Integrating Oral and Systemic Health: Innovations in Transdisciplinary Science, Health Care and Policy" (1).

As we were preparing these documents the world was facing a pandemic due to the SARSCoV2 virus, which disrupted all our lives, our directions, and our priorities in numerous and unimaginable ways, some collectively and some personally. But this time period has only heightened our collective awareness of the need to overcome the divide between medicine and dentistry to improve quality of health care for all, but especially for populations suffering disproportionately from disease impacts-whether due to the coronavirus, or long-standing oral disease inequities. Further, we partnered with the Santa Fe Group (SFG), which had similarly launched an effort addressing key medical-dental integration issues, Continuum on the Benefits of Integrating Oral Health into Healthcare, with a series of webinars, all available for viewing 
on-demand, and a virtual Salon and a virtual Salon held September (https://santafegroup.org/events/). As a long-standing SFG member and oral health advocate, Dr. Mouradian was asked to lead this effort which involved numerous scientists, clinicians, policymakers, health advocates and industry partners from multiple organizations and entities.

With the release date set to be in close proximity with the Salon, we are delighted to bring you this eBook, a partnership between the SFG and Frontiers, a collection of many peer reviewed articles resulting from the Research Topic highlighting the value of integrating oral health and disease in the context of overall health. A very brief synopsis of each article included in the Research Topic is provided below and clustered into four themes for ease of reading, realizing there is much overlap between these themes. Even as this is released, we understand there are related submissions that continue to come in, evidence of the high interest these topics have attracted. Readers are invited to check Frontiers frequently for new articles of relevance.

\section{RESEARCH/CASE REPORTS}

Several manuscripts focus on the vital need to probe the dentaloral-craniofacial history and current oral health status of patients being examined to diagnose their disease in a timely manner so treatment can be implemented in an evidence-based, efficient and timely manner. This extends to animal models where often the head is dismissed. Brenchley et al. use a case report, a patient with autoimmune polyendocrinopathy-candidiasisectodermal dystrophy (APECED), to demonstrate that dental findings can aid in early diagnosis of a disease/syndrome. In another case report by Jani et al. they discuss new findings on dental abnormalities in Loeys-Dietz Syndrome Type 1, while Lee et al. use a case report to emphasize the value of dental radiographs toward informing a diagnosis, in this situation, alter root morphology and pulp calcification identified a patient with hyperphosphatemic familial tumoral calcinosis.

Osugue et al. in a preliminary case study give evidence to support a role for periodontitis as an increased risk of stent restenosis. And in a very timely article by Cardoso et al. they demonstrate the significance of periodontal disease and other oral infections as contributors to severe respiratory disease, a hall mark consequence of SARS Cov2 infection, by providing evidence of a common mechanism of action causing hyperfunction of the immune cells. In their perspective, Integrating Dental and Medical Research Improves Oral Health, Mouradian et al. give examples where integrated research has improved health care outcomes long-term and where a lag in and/or lack of integration has resulted in fragmented and inefficient health care delivery. This theme is reinforced by a perspective article, Diabetes and Oral Health: Summary of Current Scientific Evidence for Why Transdisciplinary Collaboration Is Needed, by Borgnakke and Poudel, which emphasizes the need to avoid siloed approaches, with a specific focus on diabetes.

\section{TRANSDISCIPLINARY RESEARCH/HEALTH CARE TEAMS}

Another cluster of manuscripts brings attention to the value of research/health care collaborations, from risk assessment, to diagnosis, to treatment planning, to improves outcomes, as well as economic benefits. The studies included: (a) "Evaluating the Effectiveness of Medical-Dental Integration to Close Preventive and Disease Management Care Gaps" by Mosen et al., which suggests that medical-dental integration models facilitate the delivery of preventive and disease management services, although the authors recognize that long-term health studies are needed to strengthen their findings; (b) The perspective "Alzheimer's Disease and Oral-Systemic Health: Bidirectional Care Integration Improving Outcomes" by Rice supplies strong evidence for the need for bidirectional health care models to improve the quality of health care for our patients; and (c) The review by Chu et al. "Multiple Idiopathic Cervical Root Resorption (MICRR): A Challenge for a Transdisciplinary Medical-Dental Team" uses MICRR as a model to highlight the need for transdisciplinary teams, from basic scientists to clinicians, to inform diagnosis, treatment and clinical outcomes.

\section{DENTAL CLINICS AS PRIMARY CARE SETTINGS}

The article by Glurich et al. demonstrates that dental settings, used as a means for screening for certain diseases such as diabetes, i.e., a point-of-care model, adds value for integrative models of medical/dental management and improved compliance. A further study by Qi et al. focused on urban vs. rural dental health care systems reporting barriers to care in rural settings in China. Gordon et al. consider the value of having dentists included as primary care providers and the educational modifications, specific competencies, required to train the next generation of dentists as "Oral Physicians" or "Oral Health Primary Care Providers."

\section{EDUCATION}

Several pertinent articles discuss the importance of education and communication to inform dental-medical integration models. MacNeil and Hilario in their article "Input From Practice: Reshaping Dental Education for Integrated Patient Care" discuss the need to reshape dental education to prepare students for a healthcare environment transitioning to integrated health care models. Mays acknowledges the fact that substantial advances in health professional educational models have been developed over the last few decades, including interprofessional education and practice, but emphasizes the need to expand these models and pay more attention to models where oral health is streamlined into overall health. Expressing a boarder view of education, Kleinman et al. focus on the need to promote oral health literacy, using a systems-oriented- approach, to foster our 
unified goal of achieving oral and general health integration. Dabiri et al. provide a timely review on current data, as well research needed, to inform dental practitioners of the relationship between particle dynamics, with a focus on, but not limited to, COVID-19 transmission, and safety measures used in dental settings.

We welcome your feedback as you ready through these articles and we hope you will contribute further articles on this topic, one of much significance for our community and for advancing the quality of life for all populations.

\section{REFERENCES}

1. Mouradian W, Somerman MJ. Grand challenge: integrating oral and systemic health: innovations in transdisciplinary science, health care and policy. Front Dent Med. (2020) 1:599214. doi: 10.3389/fdmed.2020.599214

Conflict of Interest: The authors declare that the research was conducted in the absence of any commercial or financial relationships that could be construed as a potential conflict of interest.

Publisher's Note: All claims expressed in this article are solely those of the authors and do not necessarily represent those of their affiliated organizations, or those of

\section{AUTHOR CONTRIBUTIONS}

Both authors contributed equally to crafting and preparing this Editorial.

\section{ACKNOWLEDGMENTS}

Special thanks are extended to SFG and webinar, Salon contributors, Frontiers staff, chief editors and reviewers, and our Research Topic authors.

the publisher, the editors and the reviewers. Any product that may be evaluated in this article, or claim that may be made by its manufacturer, is not guaranteed or endorsed by the publisher.

Copyright (c) 2021 Somerman and Mouradian. This is an open-access article distributed under the terms of the Creative Commons Attribution License (CC BY). The use, distribution or reproduction in other forums is permitted, provided the original author(s) and the copyright owner(s) are credited and that the original publication in this journal is cited, in accordance with accepted academic practice. No use, distribution or reproduction is permitted which does not comply with these terms. 